\title{
WHEN DOES INVERSION PRESERVE CONVEXITY?
}

\author{
By D. E. Blair and J.B. Wilker
}

Dedicated to Professor Kentaro Yano on his seventieth birthday.

The study of the problem of fluid flow past a concave body in $[2,5]$ raised the question of determining which circles invert a given ellipse into a convex curve. This question was answered by one of the authors in [6] and our purpose here is to extend this work to more general convex curves and to consider the same question for the surface of a convex body in Euclidean space.

\section{The problem in curve theory}

As is often done in the theory of plane curves we take the unit normal $N$ to the curve to advance the unit tangent $T$ by $\pi / 2$. Then in the Frenet equation $d T / d s=\kappa N, s$ arc length, the curvature $\kappa$ may be positive, negative or zero and reversal of the direction of travel along the curve changes the sign of $\kappa$.
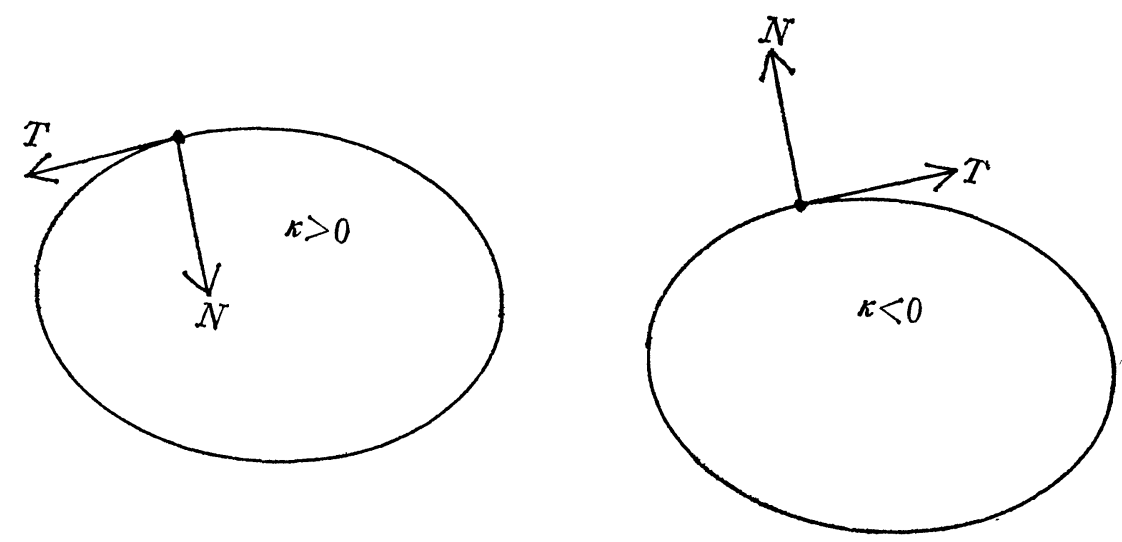

Fig. 1.

It is well known that a simple closed $C^{2}$ curve is convex if and only if $\kappa$ has constant sign.

Received August 23, 1982 
At a point where $\kappa \neq 0$, the osculating circle is well known. At a point where $\kappa=0$ the tangent line has 2 nd order contact with the curve and we call this line the "osculating circle"; moreover since we will be dealing only with convex curves its "interior" will be the half plane containing the curve.

THEOREM. Let $\mathcal{C}$ be a simple closed convex $C^{3}$ curve. A point interior to $\mathcal{C}$ is a center of inversion preserving convexity if and only if it lies in the intersection of the interiors and bonndaries of the osculating circles at the maxima of the curvature ( $C$ traversed counterclockwise). A point exterior to $\mathcal{C}$ is such a center if and only if it lies in the intersection of the exteriors and boundaries of the osculating circles at the minima of the curvature. If $\mathrm{C}$ is not a circle, inversion with center on $\mathcal{C}$ does not preserve convexity.

The main part of the proof will be to show that a point serving as a center of inversion preserving the convexity of the curve lies in the intersection of the interiors (resp. exteriors) and boundaries of all the osculating circles. The fact that it is enough to take the osculating circles at the maxima and minima of the curvature is a consequence of the following lemma of Kneser (1912) [3].

Lemma. Any osculating circle of an arc of a $C^{3}$ curve with monotonic curvature of constant sign contains every smaller osculating circle of the arc in its interior and is contained in the interior of every larger osculating circle of the arc.

Since the composition of two inversions with the same center is a dilation, which, of course, preserves convexity, the radius of the circle of inversion is immaterial in our problem. If $x_{0}$ denotes a center of inversion and $x^{*}$ the inverse of a point $x$, then inversion in the unit circle about $x_{0}$ is given by

$$
x^{*}=x_{0}+\frac{x-x_{0}}{\left|x-x_{0}\right|^{2}} .
$$

Now as $x$ traverses $\mathcal{C}$ we have the following computations.

and hence

$$
\begin{gathered}
x^{* \prime}=\frac{1}{\left|x-x_{0}\right|^{2}} T-\frac{2 T \cdot\left(x-x_{0}\right)}{\left|x-x_{0}\right|^{4}}\left(x-x_{0}\right) \\
\left|x^{* \prime}\right|=\frac{d s^{*}}{d s}=\frac{1}{\left|x-x_{0}\right|^{2}}
\end{gathered}
$$

$$
T^{*}=T-\frac{2 T \cdot\left(x-x_{0}\right)}{\left|x-x_{0}\right|^{2}}\left(x-x_{0}\right) .
$$

Differentiating this and taking the inner product with $x^{*}-x_{0}$ we have

$$
\frac{d T^{*}}{d s} \cdot\left(x^{*}-x_{0}\right)=-\frac{d T}{d s} \cdot\left(x-x_{0}\right)+2\left(\frac{\left(T \cdot\left(x-x_{0}\right)\right)^{2}}{\left|x-x_{0}\right|^{2}}-1\right) .
$$

Now with $\mathcal{C}$ being traversed counterclockwise, $\kappa \geqq 0$; thus if $x_{0}$ is interior 
to $\mathcal{C}, \kappa^{*} \geqq 0$ and the undirected angle $\alpha=\nless\left(N, x-x_{0}\right)=\varangle\left(N^{*}, x^{*}-x_{0}\right)>\pi / 2$. If $x_{0}$ is exterior to $\mathcal{C}, \kappa^{*} \leqq 0$ and $\nless\left(N, x-x_{0}\right)=\nless\left(N^{*}, x^{*}-x_{0}\right)$ though not necessarily obtuse.

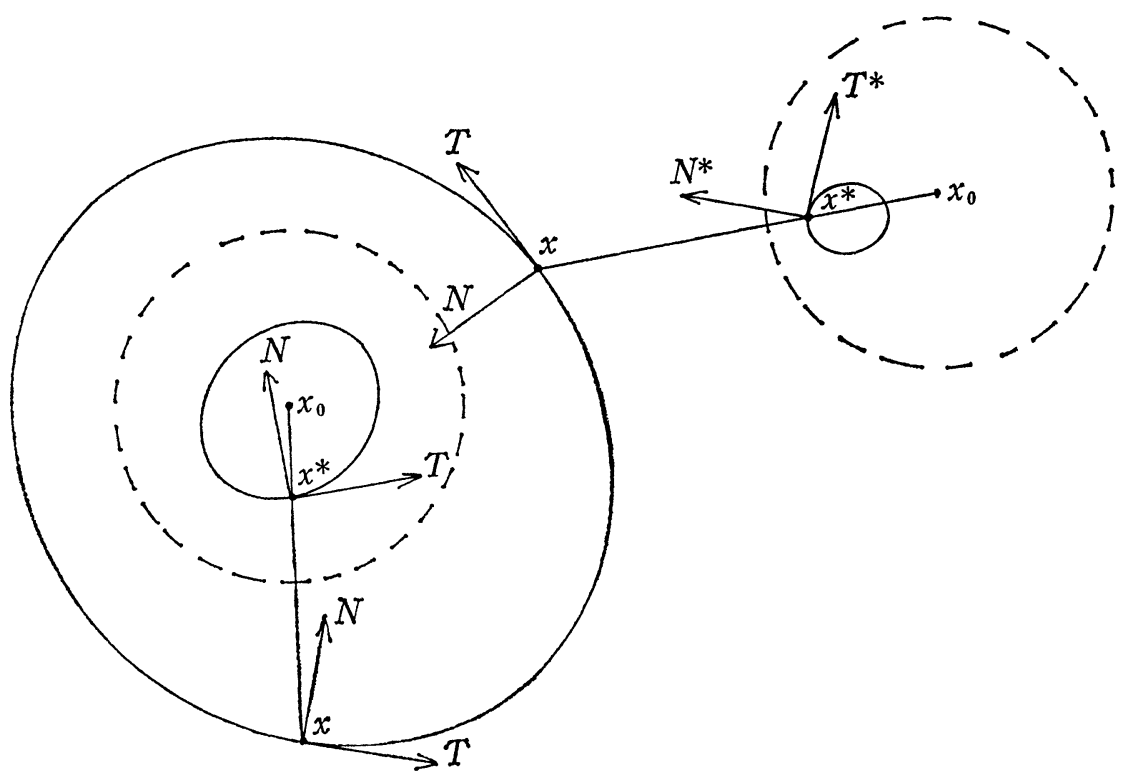

Fig. 2 .

Thus for $x_{0}$ interior to $\mathcal{C}$, (1) gives

$$
0 \geqq-\kappa\left|x-x_{0}\right| \cos \alpha+2\left(\cos ^{2}\left(\alpha-\frac{\pi}{2}\right)-1\right)
$$

i.e.,

$$
2 \cos \alpha \leqq-\kappa\left|x-x_{0}\right|
$$

or

$$
N \cdot\left(x-x_{0}\right) \leqq-\frac{\kappa}{2}\left|x-x_{0}\right|^{2} .
$$

Writing in standard cartesian coordinates $N=a i+b j, \quad x=(x, y)$ and setting $R=\frac{1}{\kappa}$ for $\kappa \neq 0$, (2) becomes

$$
\left(x_{0}-(x+R a)\right)^{2}+\left(y_{0}-(y+R b)\right)^{2} \leqq R^{2}
$$

and hence the point $x_{0}=\left(x_{0}, y_{0}\right)$ lies in or on the osculating circle at $x \in \mathcal{C}$. When $\kappa=0$, (2) becomes

$$
a\left(x_{0}-x\right)+b\left(y_{0}-y\right) \geqq 0
$$

and $x_{0}$ lies in the desired half plane. 
On the other hand if $x_{0}$ is exterior to $\mathcal{C}, \kappa^{*} \leqq 0$ and $\alpha \neq \pi / 2$ except at two points and the work below extends to these points by continuity. For $\alpha$ acute (1) yields

and for $\alpha$ obtuse

both of which yield

$$
0 \geqq-\kappa\left|x-x_{0}\right| \cos \alpha-2 \cos ^{2} \alpha
$$

$$
0 \leqq-\kappa\left|x-x_{0}\right| \cos \alpha-2 \cos ^{2} \alpha
$$

$$
2 \cos \alpha \geqq-\kappa\left|x-x_{0}\right|
$$

or

$$
N \cdot\left(x-x_{0}\right) \geqq-\frac{\kappa}{2}\left|x-x_{0}\right|^{2} .
$$

(3) is the exterior and boundary of the disk (2). Thus $x_{0}$ lies on or outside the osculating circle at $x$. Similarly when $\kappa=0$ we have the desired half plane as before.

Conversely if $x_{0}$ is a point in these intersections the reversal of the above argument shows that $x_{0}$ is a center of inversion preserving the convexity.

If $\mathcal{C}$ is a circle the set of centers of inversion preserving the convexity is the entire plane. If $\mathcal{C}$ is not a circle, inversion about any point $x_{0} \in \mathcal{C}$ destroys the convexity. For consider the osculating circle at $x_{0}$ and invert both it and the curve with center $x_{0}$; the circle inverts to a line and $\mathcal{C}$ inverts to a curve which is asymptotic to the line in both directions and is therefore not convex.

\section{The problem in surface theory}

Turning to the 3-dimensional problem we first recall that a smooth closed surface (compact without boundary) $M$ in $E^{3}$ with non-negative Gaussian curvature $K$ is convex (Chern-Lashof [1]). For $K>0$ this is a classical result of Hadamard [4].

Recall also however that a surface does not have an osculating sphere at each point, but we do have two principal curvatures $\kappa_{1}$ and $\kappa_{2}$ which for $K>0$ have the same sign. Thus we define the principal spheres at $x \in M$ to be the spheres of radius $\frac{1}{\left|\kappa_{\imath}\right|}, i=1,2, \kappa_{i} \neq 0$, centered on the normal line, tangent to $M$ at $x$ and on the side of the tangent plane containing the surface (our surfaces being convex). If one (or both) of the $\kappa_{\imath}$ 's vanish, we define the corresponding "principal sphere" to be the tangent plane and its "interior" to be the half space containing the surface.

Finally note that when the outer normal to a closed convex surface is chosen, the principal curvatures $\kappa_{\imath}$ as eigenvalues of the Weingarten map (as the linear transformation associated to the second fundamental form) are non-positive.

THEOREM. Let $M$ be a smooth closed convex surface in $E^{3}$. A point interior to $M$ is a center of inversion preserving convexity if and only if it lies in the intersection of the interiors and boundaries of all the (small) principal spheres. A 
point exterior to $M$ is such a center if and only if it lies in the intersection of the exteriors and boundaries of all the (large) principal spheres. If $M$ is not a sphere, inversion with center on $M$ does not preserve convexity.

Proof. If $M$ has a neighborhood of umbilics, the neighborhood is a piece of a sphere or plane whose inverse is again a piece of a sphere or plane. Thus we may assume that $M$ is locally umbilic free and extend to umbilics by continuity. In particular we may choose local coordinates such that the coordinate curves are lines of curvature; in turn then for the first and second fundamental forms $g$ and $h$ we have $g_{12}=h_{12}=0$. As before we denote inversion in the unit sphere about $x_{0}$ by

$$
x^{*}=x_{0}+\frac{x-x_{0}}{\left|x-x_{0}\right|^{2}} .
$$

Now as $x$ traverses $M$ we have the following computations.

$$
\begin{gathered}
x_{\imath}^{*}=\frac{1}{\left|x-x_{0}\right|^{2}} x_{i}-\frac{2 x_{i} \cdot\left(x-x_{0}\right)}{\left|x-x_{0}\right|^{4}}\left(x-x_{0}\right) \\
g_{\imath \jmath}^{*}=x_{\imath}^{*} \cdot x_{\jmath}^{*}=\frac{1}{\left|x-x_{0}\right|^{4}} g_{\imath \jmath} \\
x_{\imath \jmath}^{*}=\frac{1}{\left|x-x_{0}\right|^{2}} x_{\imath j}-\frac{2 x_{j} \cdot\left(x-x_{0}\right)}{\left|x-x_{0}\right|^{4}} x_{i}-\frac{2 x_{i} \cdot\left(x-x_{0}\right)}{\left|x-x_{0}\right|^{4}} x_{\jmath} \\
-2\left(\frac{x_{\imath j} \cdot\left(x-x_{0}\right)+x_{i} \cdot x_{\jmath}}{\left|x-x_{0}\right|^{4}}-\frac{4\left(x_{i} \cdot\left(x-x_{0}\right)\right)\left(x_{j} \cdot\left(x-x_{0}\right)\right)}{\left|x-x_{0}\right|^{6}}\right)\left(x-x_{0}\right) .
\end{gathered}
$$

Let $n$ be the unit outer normal to $M$. The inverted normal is then given by

$$
n^{*}=n-\frac{2\left(n \cdot\left(x-x_{0}\right)\right)}{\left|x-x_{0}\right|^{2}}\left(x-x_{0}\right) \text {. }
$$

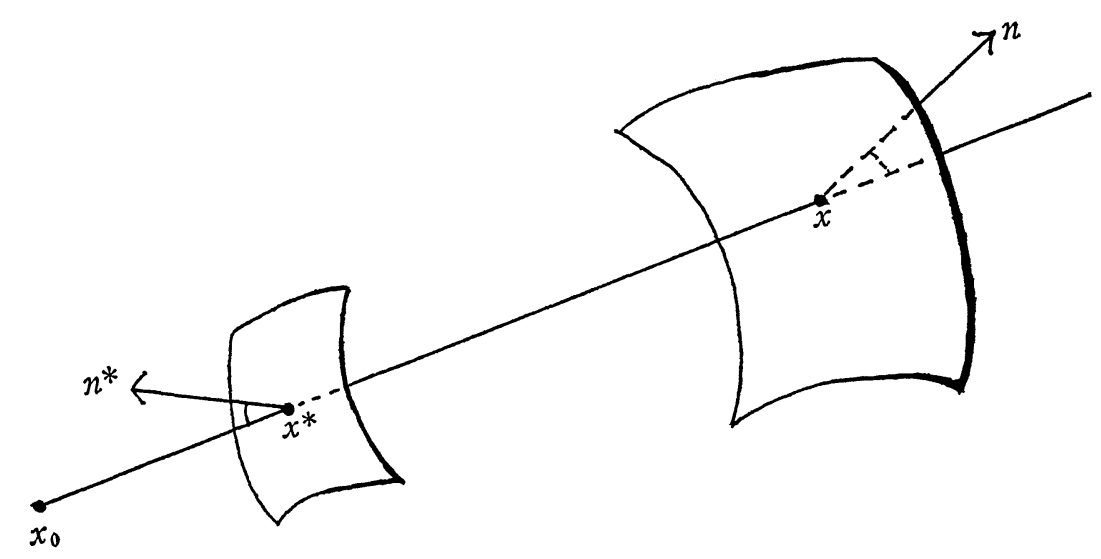

Fig. 3. 
Now in preparation for finding the second fundamental form $h^{*}$ with respect to an outer normal we have after some computation

$$
x_{\imath \jmath}^{*} \cdot n^{*}=\frac{1}{\left|x-x_{0}\right|^{2}} h_{\imath \jmath}+\frac{2 n \cdot\left(x-x_{0}\right)}{\left|x-x_{0}\right|^{-}} g_{\imath \jmath} .
$$

In particular we have $h_{12}^{*}=0$ as well as $g_{12}^{*}=0$ and the principal curvatures $\kappa_{\imath}^{*}=g^{* i i} h_{\imath \imath}^{*}$ (no sum).

If $x_{0}$ is interior to $M, n^{*}$ is the inner normal and we use $-n^{*}$ as the outer normal. Therefore for the second fundamental form

and hence

$$
h_{\imath \jmath}^{*}=-\frac{1}{\left|x-x_{0}\right|^{2}} h_{\imath j}-\frac{2 n \cdot\left(x-x_{0}\right)}{\left|x-x_{0}\right|^{4}} g_{\imath \jmath}
$$

$$
0 \geqq \kappa_{\imath}^{*}=-\kappa_{\imath}\left|x-x_{0}\right|^{2}-2 n \cdot\left(x-x_{0}\right)
$$

or

$$
n \cdot\left(x-x_{0}\right) \geqq-\frac{\kappa_{2}}{2}\left|x-x_{0}\right|^{2} .
$$

Again in cartesian coordinates, setting $n=a_{\imath}+b_{\jmath}+c k, x=(x, y, z)$ and $R_{\imath}=\frac{1}{\left|\kappa_{\imath}\right|}$ for $\kappa_{i} \neq 0$, we have

$$
\left(x_{0}-\left(x-a R_{\imath}\right)\right)^{2}+\left(y_{0}-\left(y-b R_{\imath}\right)\right)^{2}+\left(z_{0}-\left(z-c R_{\imath}\right)\right)^{2} \leqq R_{i}{ }^{2}
$$

and hence $x_{0}$ lies inside the principal spheres at $x$.

If $x_{0}$ is exterior to $M, n^{*}$ is the outer normal and we have $h_{\imath \jmath}^{*}=x_{i j}^{*} \cdot n^{*}$. Therefore

$$
-\frac{\kappa_{2}}{2}\left|x-x_{0}\right|^{2} \geqq n \cdot\left(x-x_{0}\right)
$$

which is the exterior of the principal sphere. If $\kappa_{\imath}=0$ we have the desired half spaces.

Conversely as in the curve theory case, a point in these intersections is a center of inversion preserving the convexity.

Finally if $M$ is not a sphere and $x_{0} \in M$, inversion about $x_{0}$ inverts the principal spheres to a pair of parallel planes and $M$ to a surface which is asymptotic in both directions to a line in each of the planes, so, as in the curve theory case, $M$ is not convex.

\section{REFERENCES}

[1] S. S.CHERN AHD R.K. LASHOF, On the total curvature of immersed manifolds II, Michigan Math. J. 5 (1958), 5-12.

[2] J.M. DorrepaAl, Stokes flow past a smooth cylinder, J. Engineering Math. 12 (1978), 177-185.

[3] H. Guggenheimer, Differential Geometry, McGraw-Hill, New York, 1963.

[4] J. Hadamard, Sur certaines proprietés des trajectories en dynamique, J. Math. Pures Appl. 3 (1897), 331-387. 
[5] K. B. Ranger, The Stokes flow round a smooth body with an attached vortex, J. Engineering Math. 11 (1977), 81-88.

[6] J.B. WilkeR, When is the inverse of an ellipse convex? Utilitas Math. 17 (1980), $45-50$.

Department of Mathematics Michigan State University EAST LANSing, Michigan 48824 U.S.A.
Physical Science Division

Scarborough College UNIVERSITY OF TORONTO West Hill, Ontario MIC 1A4 CANADA 